\title{
Study protocol for OptimalTTF-2: enhancing Tumor Treating Fields with skull remodeling surgery for first recurrence glioblastoma: a phase 2, multi-center, randomized, prospective, interventional trial
}

N. Mikic ${ }^{1,2^{*}}$, F. R. Poulsen ${ }^{3,4}$, K. B. Kristoffersen ${ }^{5}$, R. J. Laursen ${ }^{6}$, T. L. Guldberg ${ }^{7}$, J. Skjøth-Rasmussen ${ }^{8}$, E. T. Wong ${ }^{9,10}$, S. Møller ${ }^{11}$, R. H. Dahlrot ${ }^{12}$, J. C. H. Sørensen ${ }^{1,2}$ and A. R. Korshøj ${ }^{1,2}$

\begin{abstract}
Background: OptimalTTF-2 is a randomized, comparative, multi-center, investigator-initiated, interventional study aiming to test skull remodeling surgery in combination with Tumor Treating Fields therapy (TTFields) and best physicians choice medical oncological therapy for first recurrence in glioblastoma patients. OptimalTTF-2 is a phase 2 trial initiated in November 2020. Skull remodeling surgery consists of five burrholes, each $15 \mathrm{~mm}$ in diameter, directly over the tumor resection cavity. Preclinical research indicates that this procedure enhances the effect of Tumor Treating Fields considerably. We recently concluded a phase 1 safety/feasibility trial that indicated improved overall survival and no additional toxicity. This phase 2 trial aims to validate the efficacy of the proposed intervention.
\end{abstract}

\footnotetext{
*Correspondence: nikmik@rm.dk

'Department of Neurosurgery, Aarhus University Hospital, Palle Juul-Jensens Blvd 165, 8200 Aarhus, Denmark

${ }^{2}$ Department of Clinical Medicine, Aarhus University, Palle Juul-Jensens Blvd. 82, 8200 Aarhus, Denmark

Full list of author information is available at the end of the article
}

(c) The Author(s). 2021 Open Access This article is licensed under a Creative Commons Attribution 4.0 International License, which permits use, sharing, adaptation, distribution and reproduction in any medium or format, as long as you give appropriate credit to the original author(s) and the source, provide a link to the Creative Commons licence, and indicate if changes were made. The images or other third party material in this article are included in the article's Creative Commons licence, unless indicated otherwise in a credit line to the material. If material is not included in the article's Creative Commons licence and your intended use is not permitted by statutory regulation or exceeds the permitted use, you will need to obtain permission directly from the copyright holder. To view a copy of this licence, visit http://creativecommons.org/licenses/by/4.0/. The Creative Commons Public Domain Dedication waiver (http://creativecommons.org/publicdomain/zero/1.0/) applies to the data made available in this article, unless otherwise stated in a credit line to the data. 
Methods: The trial is designed as a comparative, 1:1 randomized, minimax two-stage phase 2 with an expected 70 patients to a maximum sample size of 84 patients. After 12-months follow-up of the first 52 patients, an interim futility analysis will be performed. The two trial arms will consist of either a) TTFields therapy combined with best physicians choice oncological treatment (control arm) or b) skull remodeling surgery, TTFields therapy and best practice oncology (interventional arm). Major eligibility criteria include age $\geq 18$ years, 1 st recurrence of supratentorial glioblastoma, Karnofsky performance score $\geq 70$, focal tumor, and lack of significant co-morbidity. Study design aims to detect a 20\% increase in overall survival after 12 months (OS12), assuming OS12 =40\% in the control group and OS12=60\% in the intervention group. Secondary endpoints include hazard rate ratio of overall survival and progression-free survival, objective tumor response rate, quality of life, KPS, steroid dose, and toxicity. Toxicity, objective tumor response rate, and QoL will be assessed every 3rd month. Endpoint data will be collected at the end of the trial, including the occurrence of suspected unexpected serious adverse reactions (SUSARs), unacceptable serious adverse events (SAEs), withdrawal of consent, or loss-to-follow-up.

Discussion: New treatment modalities are highly needed for first recurrence glioblastoma. Our proposed treatment modality of skull remodeling surgery, Tumor Treating Fields, and best practice medical oncological therapy may increase overall survival significantly.

Trial registration: ClinicalTrials.gov Identifier: NCT0422399, registered 13. January 2020.

Keywords: Craniectomy | glioblastoma | neuro-oncology | neurosurgery | tumour treating fields |

\section{Background}

Glioblastoma (GBM) is the most common primary brain cancer, while simultaneously being the most devastating in terms of overall survival (OS) and reduction of quality of life. Despite maximum safe surgical resection, radiotherapy with concomitant and adjuvant temozolomide the reported OS is in the range of $12-18$ months [1, 2]. GBM has a notoriously high recurrence rate, and the OS after first recurrence is approximately $6-10.4$ months, median progression-free survival (PFS) $1.5-2.8$ months, and overall, 12-month survival (OS12) 20-34,1\% [3] [4, 5]. Once recurrence is observed, the treatment options are limited and there is no established and effective standard of care. Novel treatments are therefore highly warranted.

Tumor Treating Fields (TTFields) is an antimitotic cancer treatment that works with low-intensity $(1-3 \mathrm{~V} /$ $\mathrm{cm})$ and intermediate frequency $(\sim 200 \mathrm{kHz})$ electric fields. TTFields is delivered noninvasively through two orthogonal pairs of transducer arrays placed on the shaved scalp. TTFields frequency is tuned for distinct cancer cell lines; as a result, tumor cells are disrupted during mitosis, while healthy quiescent cells are not negatively affected [6].

When TTFields are added to maintenance temozolomide for newly diagnosed GBM, median OS is significantly increased to 20.9 months $(\mathrm{HR}=0.63 ; 95 \% \mathrm{CI}, 0.53-0.76 ; P<$ $0.001)$ and PFS to 6.7 months $(\mathrm{HR}=0.63$; $95 \% \mathrm{CI}, 0.52-$ 0.76 ; $P<0.001$ ) [7]. In the recurrent GBM setting, a randomized trial (EF-11) showed comparable survival outcomes between TTFields monotherapy (median OS, 6.6 months) and best physician's choice chemotherapy (BPC) (median OS 6.0 months $(p=0.27)$ ). Further, the TTFields therapy group had significantly less serious adverse events and better quality of life [5]. Following the EF-11 trial, a registry study analyzed all recurrence GBM patients in the U. S treated with TTFields. (regardless of number of recurrences, KPS, previous treatment) and showed an OS of 9.6 months and OS12 at $44 \%$ for patients treated with TTFields monotherapy [8]. Currently TTFields is approved for newly diagnosed GBM and recurrent GBM.

A novel method of TTFields delivery was proposed by Korshøj et al., involving "skull remodelling surgery" (SRsurgery), i.e., craniectomy or burrholes, to create a low resistance pathway for electrical field strength during TTFields therapy. By using finite element analysis to calculate the electric field distribution in a realistic head model [9-11], preclinical research shows that removal of a standard craniotomy bone flap increases the electrical field strength by $60-70 \%$ in superficially located tumor sites and expected percentage (30-50\%) of tissue in growth arrest [12]. In addition, multiple smaller burr holes are more efficient than single craniectomies for creating increased electrical field strength of equivalent area [12].

These findings [9-12] resulted in a clinical (phase 1) single-arm pilot study. OptimalTTF-1 (NCT02893137) testing the safety and feasibility of combining "skull remodeling surgery" (SR-surgery) with TTFields therapy and BPC chemotherapy for first recurrence glioblastoma $(n=15)$. The trial concluded that the combination of SR-surgery and TTFields therapy was safe and feasible without additional toxicity and with OS at 15.5 months, OS12 at 55\%, and PFS at 4.6 months [13].

The current proposed phase 2 trial aims to validate this hypothesis in a randomized comparative setting. This manuscript is based on protocol version 2.0 dated 7th November 2020. 


\section{Methods/design}

The study is designed as an investigator-initiated, prospective, multi-center, multi-national, 1:1 randomized, minimax two-stage, comparative, phase 2 trial, investigating efficacy of the intervention [14]. Patients randomized to the control arm will receive TTFields therapy plus physician's best choice medical oncological therapy $(\mathrm{PBC})$. Patients in the interventional arm will receive SRsurgery in addition to TTFields therapy and PBC treatment. The trial will enroll an expected sample size of 70 patients from 4 sites with Aarhus University Hospital, Denmark, as the Sponsor and coordinating site. The 3 other sites in Denmark are Odense University Hospital, Aalborg University Hospital and Rigshospitalet. The primary outcome will be overall survival at 12-months (OS12). The trial is designed to detect a $20 \%$ increase in OS12 in the interventional arm compared to control (from $40 \%$ in the control arm to $60 \%$ in the interventional arm). Secondary outcomes will include PFS, quality of life (QoL), Karnofsky Performance Score (KPS), and objective response rate (ORR). Interim futility analysis will be conducted after 12 months follow-up of the first 52 patients. The trial will be stopped at interim analysis if the OS12 is equal or higher in the control arm compared to the interventional arm. The trial began in November 2020 and is expected to end in December 2023. The inclusion period is expected to be the first 24 months of the trial.

\section{Primary endpoint}

1. Overall survival rate at 12 months (OS12)

\section{Secondary endpoints}

1. Median PFS.

2. Median OS.

3. OS rate at 24 months and 36 months.

4. PFS rate at 6 months.

5. Overall objective response rate assessed by the modified RANO criteria (ORR).

6. Quality of life assessment (EORTC QLQ-C30 and QLQ-BN20).

7. Cumulative corticosteroid dosage.

8. KPS decline

9. Adverse events severity and frequency (CTCAE version 5.0).

Survival estimates will be measured from the time of inclusion.

\section{Trial overview}

\section{Screening and enrollment}

Seventy patients with first recurrence GBM according to RANO [15] criteria will be enrolled. Potential trial participants will be identified at an institutional multidisciplinary neuro-oncological tumor board and subsequently referred for enrollment and eligibility screening. All inclusion scans are assessed by a trained neuroradiologist. Immediately ineligible patients, e.g., due to poor performance status, multifocal disease, significant comorbidity, evidence of extracranial primary tumor, or other excluding circumstances, will not be referred for screening.

\section{Eligibility criteria \\ Inclusion criteria}

- age 18 years or older

- first recurrence GBM based on the RANO criteria

- whole-brain MRI according to the consensus recommendations for a standardized brain tumor imaging protocol in clinical trials not older than 4 weeks from assessment

- estimated survival $\geq 3$ months

- supratentorial tumor location

- focal disease in the vicinity of the previously known tumor or resection cavity,

- $\mathrm{KPS} \geq 70$

- Ability to comply with TTFields therapy

- Eligibility and indication for diagnostic or therapeutic neurosurgery and subsequent best practice oncological therapy

- tumor characteristics indicating significant expected benefit from feasible craniectomy or SR-surgery combined with TTFields therapy, i.e. (a) focal tumor and (b) most superficial border of tumor or resection cavity closer than $2 \mathrm{~cm}$ from the brain surface. The requirement for focal and superficially located disease is imposed to ensure an expected benefit from SR-surgery

- use of validated anti-conception for fertile female participants in concordance with guidelines provided by the Danish Health and Medicines Authority

- Signed written consent form

\section{Exclusion criteria}

- Pregnancy or nursing (fertile female participants will be required to take a validated pregnancy test for evaluation of pregnancy)

- Infra-tentorial tumor

- Implanted pacemaker, defibrillator, deep brain stimulator, other implanted electronic devices in the brain, or documented clinically significant arrhythmias

- Uncontrollable symptomatic epilepsy refractory to standard medication,

- Contraindications for skull remodeling surgery, e.g., bleeding diathesis or severe infection

- Significant co-morbidities, i.e. (a) significant liver function impairment (ALAT > 210 umol/L for men 


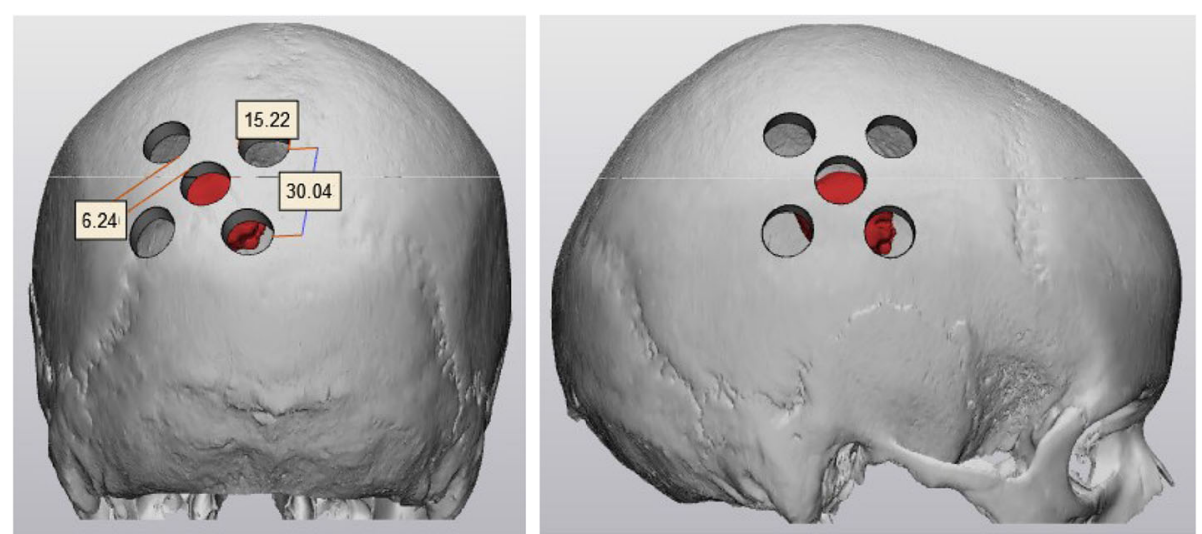

Fig. 1 SR-surgery configuration shown. The central burrhole is placed directly over the tumor or tumor resection cavity. Each of the five burrholes is $15 \mathrm{~mm}$ in diameter

and $>135 \mathrm{umol} / \mathrm{L}$ for women or total bilirubin $>25$ umol/L), (b) significant renal impairment (serum creatinine $>1.7 \mathrm{mg} / \mathrm{dL}=150 \mathrm{umol} / \mathrm{L}$ ), (c) coagulopathy (INR $>1.8$ or APTT $>57$ s), (d) thrombocytopenia (platelet count $<100 \times 103 / \mu \mathrm{L}=100 \times 270,109 / \mathrm{L})$, $(\mathrm{e})$ neutropenia $(\mathrm{ANC}<1.5 \times 103 / \mu \mathrm{L}=1.5 \times 109 / \mathrm{L})$, $(\mathrm{f})$ anemia $(\mathrm{Hb}<10 \mathrm{~g} / \mathrm{L}=6.0 \mathrm{mmol} / \mathrm{l})$

- Severe cognitive impairment

- Active participation in another therapeutic interventional clinical trial

\section{Surgery}

All trial participants will receive a maximal safe resection or tumor biopsy. This serves a dual purpose a) to confirm histopathology recurrence of GBM per $2016 \mathrm{WHO}$ criteria [16] and exclude non-eligible patients. b) to reduce trial participant risk by only performing SR surgery as an addition to biopsy or maximal safe resection.

The SR surgery has been standardized and described in a trial standard operating procedure. It will involve five burrholes, each $15 \mathrm{~mm}$ in diameter. The configuration is shown in Fig. 1. The central burrholes will be placed directly over the tumor or tumor resection cavity. The configuration is based upon simulations using realistic patient head models or virtually introduced tumors in healthy head models and various skull remodelling configurations. These simulations showed that the configuration in Fig. 1 is optimal. The details of the work performed are beyond the scope of this article but will be described in subsequent published work.

Post-operative MRI (within $72 \mathrm{~h}$ ) will be performed for all patients undergoing tumor resection to assess the extent of resection. If the extent of resection is unsatisfactory, a repeated surgical resection may be indicated if it can be performed safely [17]. Repeated surgery will thus not affect the patient's eligibility for continued participation in the trial. A new post-operative MRI will be conducted preferably within $72 \mathrm{~h}$ after repeated resection. All neuroimaging used for response assessment will comply with the consensus recommendations for a standardized brain tumor imaging protocol in clinical trials [18]. A post-operative 3D CT scan of the cranium will also be performed to assess and visualize the skull remodelling results. Toxicity, complications to surgery, and estimation of KPS will be performed postoperatively.

\section{Randomization and blinding}

Randomization (1:1 ratio) will be performed perioperatively after completion of resection or tumor biopsy to minimize that the allocation will influence the surgeon's operation intentions. Randomiation is done via a Redcap module, which is set to permuted blocks of varying sizes $(4 / 6 / 8)$ in random order across the entire trial. Each site will only have insight into their previous randomization making predictions difficult. The frequency of the various block sizes is unknown to all but the trial independent Aarhus University Redcap administrator. Once the randomization is done in Redcap it is impossible to edit. Patients will be randomized in a 1:1 ratio to receive TTFields and best practice medical oncological treatment with or without skull remodeling surgery. Local principal investigators, co-investigators, and subinvestigators will not be blinded, as optimal array planning will require knowledge about skull-remodelling surgery. The operating surgeon performing the skullremodelling surgery will not be blinded. Patients will not be blinded, as it will be possible for the patient to feel the skull configuration and thereby determine whether SR-surgery was performed. Similarly, TTFields sham treatment is not possible due to different reasons like for example the heat emanating from the arrays. 


\section{TTFields and medical oncological therapy}

Patients with confirmed recurrence of GBM will proceed to receive TTFields and PBC. TTFields therapy and PBC will be initiated between 3 and 5 weeks after surgery. A baseline MRI of the brain and a baseline clinical assessment will be performed within 4 weeks before initiation of TTFields therapy. Often, the post-operative MRI will constitute the baseline MRI. The TTFields array layout will be planned per standard operating procedures to maximize the field intensity in the region of pathology [19-21]. We will use standard settings for the Tumor Treating Fields device, i.e. a frequency of $200 \mathrm{kHz}$ and a peak-to-peak current of 1.8-2 A. TTFields will be combined with physician's best choice medical oncological therapy, such as bevacizumab, temozolomide, irinotecan, lomustine, or combinations thereof, per the EANO guidelines [7, 22, 23].

\section{Follow-up and response assessment}

During active TTFields treatment, patients will be followed regularly as per local guidelines and with clinical examinations, laboratory tests, and MRI, typically every 12 weeks or with shorter intervals. Deviations to the 12-week interval may be applied to accommodate logistical challenges or specific considerations for the individual case. Also, QoL will be assessed using QLQ-C30 and QLQ-BN20. Toxicity evaluation (CTCAEv5) will be conducted every twelve weeks. The follow-up regimen may be revised at the discretion of the treating physician. Particularly, additional follow-up including clinical and neuroradiological investigations, QoL assessments, toxicity evaluation, and neuroimaging may be done in connection with suspected or validated progression. Treatment response will be evaluated using the modified criteria for radiographic response assessment in glioblastoma clinical trials, which have been modified from the original RANO criteria to account for the potential delayed response previously observed for TTFields therapy [24, 25].

\section{Central review}

MRI scans will undergo a central retrospective review by an independent trained neuroradiologist and performed in accordance to the recent consensus recommendation for brain tumor imaging [18]. If a discrepancy is observed between the local site and central review neuroradiologist, the images will be reviewed centrally by a third independent neuroradiologist. Decisions regarding the treatment and trial eligibility of participants will be made continuously by the local investigators, based on local response assessment. The central review will serve as post-hoc data quality assurance.

\section{Sample size and statistical considerations}

The sample size calculations were based on a randomized, comparative, minimax two-stage design [14] and the binomial primary outcome of OS12 for the intent to treat trial participants per protocol, i.e. for whom TTFields therapy was initiated. This means that patients excluded prior to TTFields therapy are not included in the primary outcome analysis. Therefore, additional patients may be enrolled to ensure that the planned sample receives active TTFields treatment. The trial aims to indicate whether the intervention is superior to control and thus worthy of further phase 3 investigation. The expected OS12 in the control arm is set to $40 \%$ based on the EORTC 26101 trial [3], in which patients with first recurrence of GBM were treated with lomustine monotherapy or lomustine/bevacizumab combination therapy. The trial showed an OS12 of approximately 30\%. We therefore set the expected level of OS12 to be slightly above this threshold given the fact that TTFields is added to the treatment, which otherwise represents recommended practice. No current studies provide accurate OS12 estimates for a population comparable to the study control arm. Therefore, we based the estimate on an expected benefit of $10 \%$ from TTFields alone compared to EORTC 26101. Setting the probabilities of false-positive and false-negative trial results to $\alpha=0.15$ and $1-\beta=0.80$, respectively, and defining the expected target level of OS12 to be OS12 $=0.6$, i.e. a $31620 \%$ absolute increase (and 50\% relative increase) compared to the control arm, we calculated a maximum sample size of $n=42$ patients in each arm (total $n=84$ ) and an expected total sample size (en $=69.8)$. The expected sample size is calculated as en $=\mathrm{n} 1 \times$ PET $0+\mathrm{n} \times(1-$ PET0 $)$, where $\mathrm{n} 1=$ 52 is the collective sample size of both groups at interim (stage 1 ) analysis, PET0 $=0.44$ is the probability of early futility termination. The numbers are based on the given statistical parameters and a two-stage minimax design as given in SH Jung 2018 [26]. Interim futility analysis will be conducted after endpoint assessment of 52 patients and the trial will be terminated if the experimental arm performs worse than the control arm. In this case, the futility criterion will be defined as OS12experimental < OS12 control, and this conclusion will determine futility at marginal power and significance levels of $1-\beta^{*}=0.80$ and of $\alpha^{*}=0.20$, respectively. If the trial is not terminated at interim stage 1 analysis, the trial will proceed to enroll a total of 84 patients and terminate when the final OS12 endpoint data have been obtained for all patients. The secondary outcome measures of hazard rate ratios of PFS and OS will be tested using the log-rank test at the 0.05 alpha level. Time-to-event endpoints will be estimated from the time of inclusion. Toxicity and adverse events will be reported using absolute numbers and appropriate risk estimates. Final analysis will be conducted 
when all patients have been excluded or censored, e.g. due to end-of-trial or loss-to-follow-up. Subgroup analysis based on prognostic factors will be performed, incl. Analysis of patient characteristics in each arm and identification of characteristics of potential responders to the intervention. This will also include a correlation analysis between the calculated field distribution and outcome estimates.

\section{Withdrawal from study}

The study will be terminated when all enrolled participants have been excluded from the trial and the necessary data have been acquired. A patient may be excluded from the trial due to one of the following reasons:

- Non-GBM diagnosis provided by tumor resection/ biopsy

- Death,

- Loss to follow-up,

- End-of-trial,

- Enrollment in another interventional clinical trial,

- Withdrawal of consent

- The patient is no longer suitable for further participation due to ethical or medical safety reasons determined by the investigator.

\section{Data monitoring committee}

An Independent Data Monitoring Committee (DMC) will be created with the main purpose of patient safety. The DMC will achieve this by monitoring ongoing data, especially regarding adverse events and analyzing the benefit vs risk ratio. Furthermore, they will provide an independent scientific review of the interim analysis and recommend continuation or discontinuation of the trial. If the trial passes interim analysis, the DMC will review the final data as well. The DMC will serve in an advisory capacity to the sponsor. Members of the committee will include two clinicians with neuro-oncological trial experience, a statistician, a nurse and two laypeople.

\section{Trial steering committee}

The Trial Steering Committee (TSC) will consist of the sponsor-investigator and two representatives from each study site, in addition to one independent clinician outside of Denmark with neuro-oncological trial experience. The TSC will act in an advisory capacity to the sponsor in terms of reviewing the progress of the trial and, if necessary, recommend amendments to the protocol or trial logistics to ensure optimal trial progress. Furthermore, the recommendations provided by the DMC will be discussed and, if needed, implemented by the TSC.

\section{Publication}

All results will be published in peer-reviewed international scientific journals and presented at international scientific conferences, regardless of academic conclusions. Positive, negative, and inconclusive results will be publicly available.

\section{Discussion}

With no standardized treatment for recurrence glioblastoma and a poor prognosis, new treatment modalities or methods are highly needed. We present a randomized, interventional, clinical phase 2 trial testing a new and innovative intervention, skull remodeling surgery, to enhance TTFields therapy for first recurrence glioblastoma. Since the control arm uses a multimodal approach with TTFields therapy + BPC, we believe both trial arms are expected to show a survival benefit, regardless of randomization, compared to historically studies using either BPC or TTFields monotherapy. However, the interventional arm may benefit even greater if our phase 1 trial 15.5 months overall survival is any indication of the potential enhanced effect of SR-surgery.

All patients included would have undergone a tumor biopsy or resection, regardless of trial involvement. This reduces the risk of SR surgery to a minimum and strengthens the trial by a) reducing one statistical variable by not including patients where surgery is not indicated and b) ensuring all trial participants have a confirmed glioblastoma recurrence.

The limitations of the study are a very selected first recurrence GBM patient group, i.e. KPS $\geq 70$, no significant comorbidity, surgery indicated, intact cognition, and no multifocality.

Another limitation is that the trial participants will not be stratified when randomized, which might give unbalanced baseline characteristics and if not adjusted give misleading results. However, with the interim analysis set at 52 patients and final data not to exceed 84 patients, there are too few trial participants to sensibly implement randomization based on MGMT status, degree of resection at primary and recurrence surgery, age, KPS and steroid usage at inclusion. There is a high risk that the two arms sample sizes will be imbalanced when trying to use block randomization with too many prognostic factors and too few trial participants. Ultimately, we decided not to stratify, which might give results that are incomparable. However, we are prepared to do retrospective stratification analysis if the baseline characteristics are unbalanced, in an attempt to gain meaningful data.

Finally, due to the nature of the surgery and how the TTFields device functions, blinding of the surgeons or patients to the intervention is not possible nor a trial design including a sham device or surgery. 


\section{Abbreviations}

KPS: Karnofsky performance score; OS12: Overall survival after 12 months; SUSAR: Suspected unexpected serious adverse reactions; SAE: Serious adverse events; GBM: Glioblastoma; OS: Overall survival; PFS: Progression-free survival; TTFields / TTF: Tumor Treating Fields; SR-surgery: Skull remodeling surgery; PBC: Physician's best choice medical oncological therapy: QoL: Quality of life; KPS: Karnofsky Performance Score; ORR: Objective response rate; DMC: Independent Data Monitoring Committee; TSC: The Trial Steering Committee; GCP: Good Clinical Practice

\section{Acknowledgements}

We sincerely thank the neurosurgical and oncological department heads at Rigshospitalet, Aarhus-, Odense- and Aalborg University Hospital for their support of the trial.

Furthermore, we would sincerely like to thank the following individuals: Søren Ole Stigaard Cortnum, MD, for helping with managing the trial and recruitment of patients

Marianne Esbjerg from the Good Clinical Practice Unit, Aarhus University for monitoring the trial with dedication.

Rikke Beese Dalby, MD, PhD and Christian Rahbek, MD for participating and helping us organize the infrastructure of the central review

Karen Risgaard and Hanne Lisby from the Danish Brain Tumor Association for their support and Hanne Lisby's participation in the DMC as a layperson Jakob Hjort for creating the randomization module and troubleshooting the Redcap database

\section{Authors' contributions}

Manuscript original draft: N.M. and A.R.K. Manuscript revision and review: All. Funding acquisition: N.M. and A.R.K. Designing and modifying the protocol: All. The author(s) read and approved the final manuscript.

\section{Funding}

OptimalTTF-2 is funded by Novocure, Aarhus University, Danish Cancer Society, and Independent Research Fund Denmark. The trial protocol was designed by sponsor-investigator Aarhus University Hospital and modified by the Trial Steering Committee.

The funding bodies have peer reviewed the trial protocol but have had no role in the design of the study, analysis, and interpretation of data nor in the writing of this or future manuscripts.

\section{Availability of data and materials}

Not applicable.

\section{Declarations}

\section{Ethics approval and consent to participate}

OptimalTTF-2 (NCT04223999) will comply with international guidelines Good Clinical Practice (ICH-GCP and DS/EN ISO 14155:2012) and be monitored by the GCP unit at Aarhus University. AE reporting procedures will comply with Guidelines on medical devices. Clinical investigations: Serious adverse event reporting (MEDDEV 2.7/3) and Reporting of adverse reactions in clinical trials. All data will be handled in accordance with The Danish Act on Processing of Personal Data. Clinical trial approval has been gained from the Central Denmark Region Committee of Health Research Ethics, The Danish Data Protection Agency, and the Danish Health Authorities for all sites. All patients will receive oral and written information by a certified neurosurgeon with time to consider enrollment in the trial. Based upon the written and oral information, they will give signed informed consent.

\section{Consent for publication}

Not applicable.

\section{Competing interests}

None of the authors have any competing interests.

\section{Author details}

${ }^{1}$ Department of Neurosurgery, Aarhus University Hospital, Palle Juul-Jensens Blvd 165, 8200 Aarhus, Denmark. ${ }^{2}$ Department of Clinical Medicine, Aarhus University, Palle Juul-Jensens Blvd. 82, 8200 Aarhus, Denmark. ${ }^{3}$ Department of Neurosurgery, Odense University Hospital, Kløvervænget 47, 5000 Odense, Denmark. ${ }^{4}$ Clinical Institute BRIDGE (Brain Research InterDisciplinary Guided
Excellence), University of Southern Denmark, Winsløwparken 19, 5000 Odense, Denmark. ${ }^{5}$ Department of Oncology, Aarhus University Hospital, Palle Juul-Jensens Blvd. 99, 8200 Aarhus, Denmark. ${ }^{6}$ Department of Neurosurgery, Aalborg University Hospital, Hobrovej 18-22, 9000 Aalborg, Denmark. ${ }^{7}$ Department of Oncology, Aalborg University Hospital, Hobrovej 18-22, 9000 Aalborg, Denmark. ${ }^{8}$ Department of Neurosurgery, Rigshospitalet, Inge Lehmanns Vej 6, 2100 København $\varnothing$, Denmark. ${ }^{9}$ Beth Israel Deaconess Medical Center, 330 Brookline Ave, Boston, MA 02215, USA. ${ }^{10}$ Harvard Medical School, 25 Shattuck St, Boston, MA 02115, USA. "11Department of Oncology, Rigshospitalet, Blegdamsvej 9, 2100 København Ø, Denmark. ${ }^{12}$ Department of Oncology, Odense University Hospital, Kløvervænget 19, 5000 Odense, Denmark.

Received: 10 July 2021 Accepted: 18 August 2021

Published online: 09 September 2021

\section{References}

1. Becker KP, Yu J. Status quo--standard-of-care medical and radiation therapy for glioblastoma. Cancer J. 2012;18(1):12-9. https://doi.org/10.1097/PPO. 0b013e318244d7eb.

2. Hegi ME, Diserens AC, Gorlia T, Hamou MF, de Tribolet N, Weller M, et al. MGMT gene silencing and benefit from temozolomide in glioblastoma. N Engl J Med. 2005;352(10):997-1003. https://doi.org/10.1056/NEJMoa043331.

3. Wick W, Gorlia T, Bendszus M, Taphoorn M, Sahm F, Harting I, et al. Lomustine and Bevacizumab in Progressive Glioblastoma. N Engl J Med. 2017;377(20):1954-63. https://doi.org/10.1056/NEJMoa1707358.

4. Yung WK, Albright RE, Olson J, Fredericks R, Fink K, Prados MD, et al. A phase II study of temozolomide vs. procarbazine in patients with glioblastoma multiforme at first relapse. Br J Cancer. 2000;83(5):588-93. https://doi.org/10.1054/bjoc.2000.1316.

5. Stupp R, Wong ET, Kanner AA, Steinberg D, Engelhard H, Heidecke V, et al. NovoTTF-100A versus physician's choice chemotherapy in recurrent glioblastoma: a randomised phase III trial of a novel treatment modality. Eur J Cancer. 2012;48(14):2192-202. https://doi.org/10.1016/j.ejca.2012.04.011.

6. Joshua Lantos RJY, Miranda PC, Wenger C, Wong ET. In: Newton HB, editor. Handbook of Neuro-Oncology Neuroimaging (Second Edition): Academic Press; 2016.

7. Stupp R, Taillibert S, Kanner A, Read W, Steinberg D, Lhermitte B, et al. Effect of tumor-treating fields plus maintenance Temozolomide vs maintenance Temozolomide alone on survival in patients with glioblastoma: a randomized clinical trial. JAMA. 2017;318(23):2306-16. https://doi.org/10.1 001/jama.2017.18718.

8. Mrugala MM, Engelhard HH, Dinh Tran D, Kew Y, Cavaliere R, Villano JL, et al. Clinical practice experience with NovoTTF-100A ${ }^{\text {TM }}$ system for glioblastoma: the patient registry dataset (PRiDe). Semin Oncol. 2014; 41(Suppl 6):S4-s13. https://doi.org/10.1053/j.seminoncol.2014.09.010.

9. Korshoej AR, Mikic N, Hansen FL, Saturnino GB, Thielscher A, Bomzon Z. Enhancing tumor treating fields therapy with skull-remodeling surgery. The Role of Finite Element Methods in Surgery Planning. Annu Int Conf IEEE Eng Med Biol Soc. 2019;2019:6995-7.

10. Wenger C, Miranda PC, Salvador R, Thielscher A, Bomzon Z, Giladi M, et al. A review on tumor-treating fields (TTFields): clinical implications inferred from computational modeling. IEEE Rev Biomed Eng. 2018;11:195-207. https:// doi.org/10.1109/RBME.2017.2765282.

11. Korshoej AR, Thielscher A. Estimating the intensity and anisotropy of tumor treating fields Jsing singular value decomposition. Towards a More Comprehensive Estimation of Anti-tumor Efficacy. Annu Int Conf IEEE Eng Med Biol Soc. 2018;2018:4897-900. https://doi.org/10.1109/EMBC.2018. 8513440.

12. Korshoej AR, Saturnino GB, Rasmussen LK, von Oettingen G, Sørensen JC, Thielscher A. Enhancing predicted efficacy of tumor treating fields therapy of glioblastoma using targeted surgical Craniectomy: a computer modeling study. PLoS One. 2016;1 1(10):e0164051. https://doi.org/10.1371/journal.pone. 0164051.

13. Korshoej AR, Lukacova S, Lassen-Ramshad Y, Rahbek C, Severinsen KE, Guldberg TL, et al. OptimalTTF-1: Enhancing tumor treating fields therapy with skull remodeling surgery. A clinical phase I trial in adult recurrent glioblastoma. Neurooncol Adv. 2020;2(1):vdaa121.

14. Simon R. Optimal two-stage designs for phase II clinical trials. Control Clin Trials. 1989;10(1):1-10. https://doi.org/10.1016/0197-2456(89)90015-9. 
15. Wen PY, Macdonald DR, Reardon DA, Cloughesy TF, Sorensen AG, Galanis E, et al. Updated response assessment criteria for high-grade gliomas: response assessment in neuro-oncology working group. J Clin Oncol. 2010; 28(11):1963-72. https://doi.org/10.1200/JCO.2009.26.3541.

16. Louis DN, Perry A, Reifenberger G, von Deimling A, Figarella-Branger D, Cavenee WK, et al. The 2016 World Health Organization classification of tumors of the central nervous system: a summary. Acta Neuropathol. 2016; 131(6):803-20. https://doi.org/10.1007/s00401-016-1545-1.

17. Yong RL, Lonser RR. Surgery for glioblastoma multiforme: striking a balance. World Neurosurg. 2011;76(6):528-30. https://doi.org/10.1016/j.wneu.2011.06.053.

18. Ellingson BM, Bendszus M, Boxerman J, Barboriak D, Erickson BJ, Smits M, et al. Consensus recommendations for a standardized brain tumor imaging protocol in clinical trials. Neuro-Oncology. 2015;17(9):1188-98.

19. Korshoej AR, Hansen FL, Mikic N, von Oettingen G, JCH S, Thielscher A. Importance of electrode position for the distribution of tumor treating fields (TTFields) in a human brain. Identification of effective layouts through systematic analysis of array positions for multiple tumor locations. PLoS One. 2018;13(8):e0201957.

20. Korshoej AR, Hansen FL, Thielscher A, von Oettingen GB, Sørensen JCH. Impact of tumor position, conductivity distribution and tissue homogeneity on the distribution of tumor treating fields in a human brain: a computer modeling study. PLoS One. 2017;12(6):e0179214. https://doi.org/10.1371/ journal.pone.0179214.

21. Korshoej AR, Hansen FL, Mikic N, Thielscher A, von Oettingen GB, Sørensen $\mathrm{JCH}$, et al. Guiding principles for predicting the distribution of tumor treating fields in a human brain: a computer modeling study investigating the impact of tumor position, conductivity distribution and tissue homogeneity. Neurooncol. 2017;19(Suppl 6):vi73-vi.

22. Weller M, van den Bent M, Tonn JC, Stupp R, Preusser M, Cohen-JonathanMoyal E, et al. European Association for Neuro-Oncology (EANO) guideline on the diagnosis and treatment of adult astrocytic and oligodendroglial gliomas. Lancet Oncol. 2017;18(6):e315-e29. https://doi.org/10.1016/S1470-2 045(17)30194-8.

23. Dansk Neuro Onkologisk Gruppe. Retningslinjer for behandling af intrakranielle gliomer hos voksne. 2016.

24. Ellingson BM, Wen PY, Cloughesy TF. Modified criteria for radiographic response assessment in glioblastoma clinical trials. Neurotherapeutics. 2017; 14(2):307-20. https://doi.org/10.1007/s13311-016-0507-6.

25. Wong ET, Lok E, Swanson KD, Gautam S, Engelhard HH, Lieberman F, et al. Response assessment of NovoTTF-100A versus best physician's choice chemotherapy in recurrent glioblastoma. Cancer Med. 2014;3(3):592-602. https://doi.org/10.1002/cam4.210

26. University D. Randomized Phase II Clinical Trials using Fisher's Exact Test. Paper 7. In: Duke Biostatistics and Bioinformatics (B\&B) Working Paper Series; 2010

\section{Publisher's Note}

Springer Nature remains neutral with regard to jurisdictional claims in published maps and institutional affiliations.

Ready to submit your research? Choose BMC and benefit from:

- fast, convenient online submission

- thorough peer review by experienced researchers in your field

- rapid publication on acceptance

- support for research data, including large and complex data types

- gold Open Access which fosters wider collaboration and increased citations

- maximum visibility for your research: over $100 \mathrm{M}$ website views per year

At BMC, research is always in progress.

Learn more biomedcentral.com/submissions 